\title{
Milliliter per Cage per Week
}

National Cancer Institute

\section{Source}

National Cancer Institute. Milliliter per Cage per Week. NCI Thesaurus. Code C73752.

A dose calculation unit expressed in milliliter(s) per cage expressed per period of time equal to seven days. 